\title{
„Erlanger Broncho-Trainer“ - Neuentwicklung eines Trainingsmodells mit physiologischer Ventilation für die interventionelle Bronchoskopie
}

\author{
M. Neumann ${ }^{1}$ \\ D. Adler ${ }^{4}$ \\ T. Felzmann ${ }^{1}$ \\ J. H. Ficker ${ }^{3}$ \\ M. Wagner ${ }^{3}$ \\ W. Hohenberger ${ }^{4}$ \\ I. Schneider ${ }^{4}$ \\ R. Dierkesmann²
}

\author{
"Erlangen Broncho-Trainer" - Newly Developed Training Model with \\ Physiological Ventilation for Interventional Bronchoscopy
}

\section{Zusammenfassung}

Hintergrund: Bio-Simulationsmodelle haben sich bei zahlreichen Trainingskursen für endoskopische Eingriffe und Interventionen bewährt und spielen eine wichtige Rolle bei der Aus- und Weiterbildung, sowie bei der Einführung neuer Techniken. Auf dem Gebiet der interventionellen Bronchoskopie erhöht sich die Realität, wenn das biologische Lungengewebe nicht nur kollabiert zur Verfügung steht, sondern sich physiologisch entfaltet. Material und Methode: Auf der Basis des bekannten Erlanger Endo-Trainers kann als neue Modellmodifikation mit einem transparenten Modelldeckel ein luftdichter Abschluss der Thoraxhöhle erreicht und darin mittels einer integrierten Vakuumpumpe ein Unterdruck erzeugt werden. Damit ist es möglich, eine Trainingslunge vom Schwein vollständig zur Entfaltung zu bringen und mit einstellbaren intermittierenden Inspirationsund Exspirationsphasen „physiologisch ventilieren“ zu lassen. Die „atmende“ und vollständig entfaltbare biologische Trainingslunge vom Schwein verbessert nicht nur die anatomische Orientierung durch die vollständige dreidimensionale Entfaltung der Lunge, sondern bietet auch mit überzeugender und gewebsechter Realität eine gute Übungsmöglichkeit für verschiedene bronchoskopische Interventionen. Schlussfolgerungen: Mit diesem neuen System konnten in einem ersten Trainingskurs neben diagnostischen Techniken wie BAL (bronchoalveoläre Lavage), transbronchiale Nadelaspiration (TBNA) und periphere Zangenbiopsien unter Durchleuchtungskontrolle auch erste interventionelle Techniken wie Fremdkörperentfernung und Koagulationsverfahren erfolgreich durchgeführt werden. Diese ersten positi-

\section{Abstract}

Background: Biosimulation models have proved their value in numerous training courses for endoscopic procedures and play an important role in education and further training, as well as in the introduction of new techniques. In the field of interventional bronchoscopy, the level of realism is increased when biological lung tissue is available not only in a collapsed state, but also with physiological expansion. Methods/results: A new modification of the well-known Erlangen Endo-Trainer has been developed, involving a transparent cover that provides airtight closure of the thoracic cavity. An integrated vacuum pump creates low pressure in the interior of the model. This allows a training lung from the pig to be fully expanded and "physiologically ventilated", with adjustable intermittent inspiration and expiration phases. The breathing and completely expandable biological training lung from the pig not only provides better anatomical orientation thanks to the lung's full three-dimensional expansion, but with the convincing and realistic quality of the tissue it also provides a good practice facility for various types of bronchoscopic intervention. Conclusions: In an initial training course, the new system successfully allowed various diagnostic techniques to be practiced, such as bronchoalveolar lavage (BAL), transbronchial needle aspiration (TBNA), and peripheral forceps biopsies with transillumination guidance and interventional techniques such as foreign-body removal and electrocautery as well. These initial positive results are also encouraging for the future provision of training facilities in additional interventional techniques.

Institutsangaben

${ }^{1}$ Erlanger Chirurgie und Endoskopie Training Center

${ }^{2}$ Klinik Schillerhöhe, Pneumologie

${ }^{3}$ Klinikum Nürnberg, Medizinische Klinik 3 - Pneumologie, Allergologie, Somnologie

${ }^{4}$ Chirurgische Universitätsklinik Erlangen

Korrespondenzadresse

Dr. med. Martin Neumann · ECE-Training Center · Henkestraße 91 · 91052 Erlangen ·

E-mail: martin.neumann@endo-trainer.de ·www.ece-erlangen.de

Eingang: 26. Februar 2004 - Nach Revision alkzeptiert: 17. Oktober 2004

Bibliografie

Pneumologie 2005; 59: 18-21 @ Georg Thieme Verlag KG Stuttgart · New York

DOI $10.1055 / \mathrm{s}-2004-830136$

ISSN 0934-8387 
ven Erfahrungen haben auch die Perspektive für weitere interventionelle Techniken als Vorteil dieses neuen Trainingmodells deutlich gemacht.

\section{Hintergrund}

Der Erlanger Endo-Trainer ist als Bio-Simulationsmodell in Kombination mit speziell vorbereiteten, biologischen Präparaten seit Jahren bei praxisorientierten endoskopischen Fortbildungsveranstaltungen in der Gastroenterologie und Chirurgie im Einsatz. Dabei hat vor allem bei den interventionellen Techniken der lebensechte Eindruck und das echte Gewebe überzeugt und ein breites Spektrum von therapeutischen Eingriffen ermöglicht $[1,2]$.

Der Erlanger Endo-Trainer besteht in seiner Grundkonzeption aus einem anatomisch geformten Körper mit Kopf und ist in einer speziellen Haltevorrichtung in der gewünschten Körperdrehung fixierbar. Integriert in die Grundplatte des Simulationsmodells ist ein elektronisch geregeltes Pumpsystem, mit dem eine akute Blutung simuliert und der „Herzschlag des Patienten“ reguliert werden kann. In einer modifizierten Version ist der Modelldeckel aus einem transparenten Material geformt, der einen intraabdominellen und intrathorakalen „Kontrollblick“ erlaubt eine Hilfe, die gerade für den Anfänger wichtig ist, um nicht die Orientierung des Endoskopes, bzw. der Endoskopspitze bei den ersten Untersuchungsschritten zu verlieren. Je nach Trainingsprogramm wird in den Modellkörper ein speziell vorbereitetes Präparat vom Schwein (z.B. Ösophagus und Magen) in anatomisch korrekter Position fixiert. Die Konnektion des biologischen Gewebes mit den Kunststoffteilen des Kopf-Modells erfolgt knapp unterhalb des Larynx. Das Endoskop kann also wie beim Patienten in den Mund oder die Nase eingeführt werden und gleitet z. B. beim Gastroskopiemodell durch den Rachen am Kehlkopf vorbei in den proximalen Ösophagus. Stimmlippen und Tracheaöffnung sind bei dem Gastroskopiemodell ebenfalls zur Orientierung vorhanden.

Prinzipiell eignet sich der Erlanger Endo-Trainer auch für die Bronchoskopie, da in das Trainingsmodell auch ein Lungenpräparat nach spezieller Vorbereitung implantiert werden kann und die entsprechenden Anschlüsse vorhanden sind. Erste Trainingskurse und Interventionen wurden bereits in dieser Version durchgeführt [3]. Der Nachteil war dabei jedoch, dass die Schweinelunge in dieser ersten Modellkonfiguration ohne Vakuumsystem komplett kollabiert war und damit Realität und Untersuchungs-/Interventionsmöglichkeiten eingeschränkt waren.

Simulationsmodelle spielen eine wichtige Rolle in der Aus- und Weiterbildung, sowie bei der Einführung neuer Techniken oder bei der Durchführung experimenteller Studien, und können dabei bis zu einer gewissen Grenze den Patienten ersetzen. Bekannt sind verschiedene Modellarten, wie beispielsweise anfänglich einfache Kunststoffmodelle, oder in jüngster Zeit auch virtuelle Computersimulationsmodelle. Tiermodelle bieten eine realistische Gewebereaktion, bzw. Gewebegefühl, das vor allem bei interventionellen Eingriffen wichtig ist. Diesen Vorteil bieten auch so genannte Bio-Simulationsmodelle, bei denen einzelne präparierte Tierorgane in ein Körpermodell implantiert werden.
Von den Tierorganen eignen sich Präparate vom Schwein am besten; speziell auch die Schweinelunge mit einer dem Menschen ähnlichen Anatomie ist in diesem Zusammenhang schon länger bekannt [4-11].

Ziel der vorliegenden Arbeit ist es nun, die Realitätsnähe des Erlanger Endo-Trainers in Kombination mit einem Schweinelungenpräparat für bronchoskopische Eingriffe zu verbessern und eine Möglichkeit zu realisieren, die Trainingslunge während eines Übungseingriffes zur Entfaltung zu bringen und physiologisch ventilieren zu lassen.

\section{Material und Methoden}

\section{Ganz-Körper-Modell}

Realisiert wird diese Aufgabe mit dem an sich bekannten und eingangs kurz beschriebenen Erlanger Endo-Trainer, einem BioSimulationsmodell, das in der Basis-Version aus einem anatomischen Ganz-Körper-Modell mit Kopf besteht, und eine anatomisch nachgebildete Abdominalhöhle und Thoraxhöhle aufweist, beide von einer Zwerchfellplatte getrennt. Nach der Vorbereitung und Implantation eines Trainingspräparates in das Modell ist dieses mit einem transparenten Modelldeckel abdeckbar (Abb.1). Für die Bronchoskopie ist in das Modell an einen kranialen Stutzen als Verlängerung der Kunststofftrachea des Modellkopfes ein biologisches Lungenpräparat vom Schwein luftdicht konnektiert. Das Herz und die großen Gefäße wurden vor der Implantation in das Modell vorsichtig abpräpariert, ohne dass dabei das Lungenparenchym verletzt wurde (Abb. 2).

\section{Abdichtung}

Der Modelldeckel, der das Abdomen und die Thoraxhöhle nach ventral abdeckt, ist mit einer Gummidichtung versehen, die einen luftdichten Verschluss der Thoraxhöhle ermöglicht, nachdem das Lungenpräparat implantiert wurde und die Trachea des Präparates an den entsprechenden kranialen Modellstutzen angeschlossen wurde (Abb. 3). Das Lumen der Kunststofftrachea des Modellkopfes wird über diesen kranialen Modellstutzen in

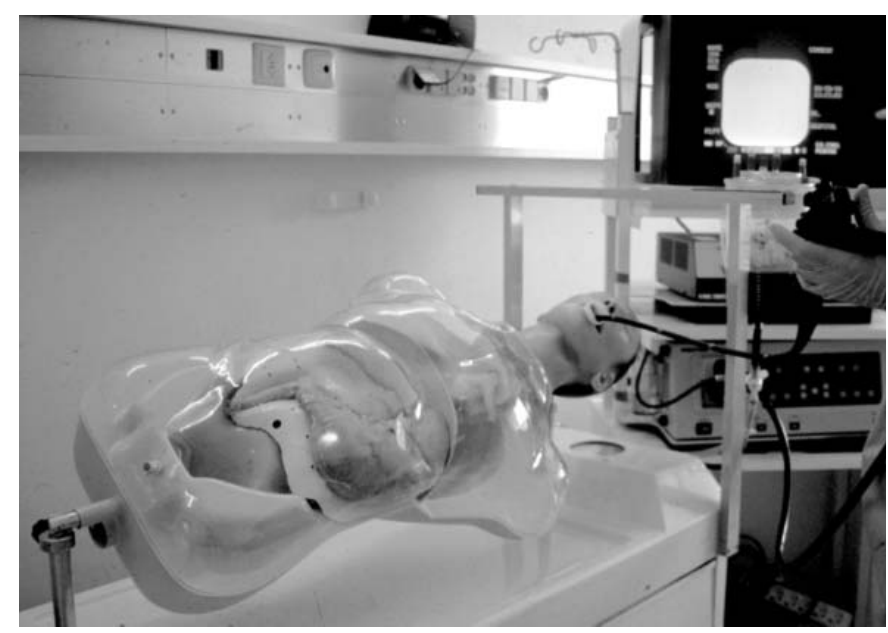

Abb. 1 Erlanger Endo-Trainer, der sich bei zahlreichen EndoskopieWorkshops in der Gastroenterologie und Chirurgie mit einem breiten Spektrum von diagnostischen und interventionellen Trainingsmöglichkeiten bewährt hat. 


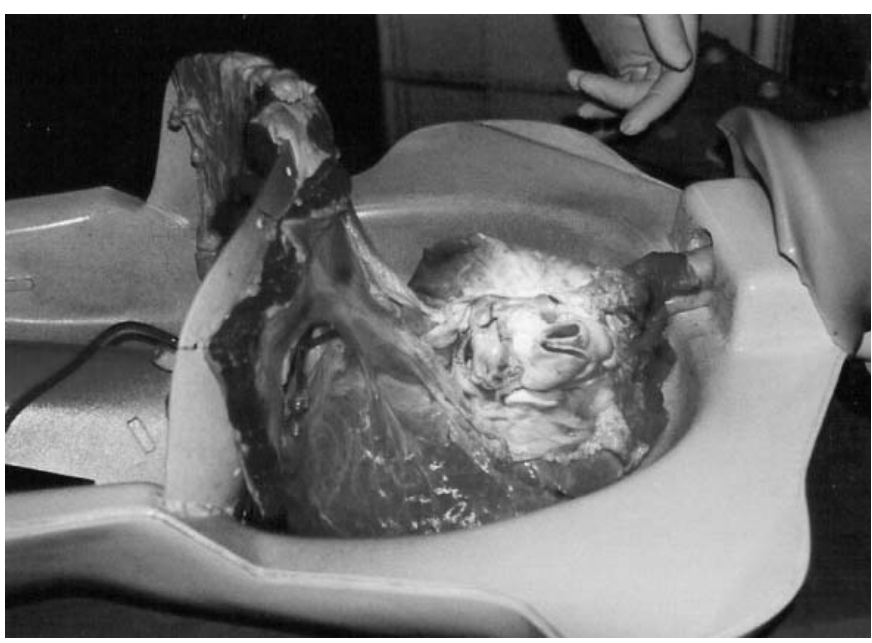

Abb. 2 Erlanger Endo-Trainer vorbereitet für die Bronchoskopie mit kollabiertem Schweinelungenpräparat. Herz und große Gefäße wurden entfernt.

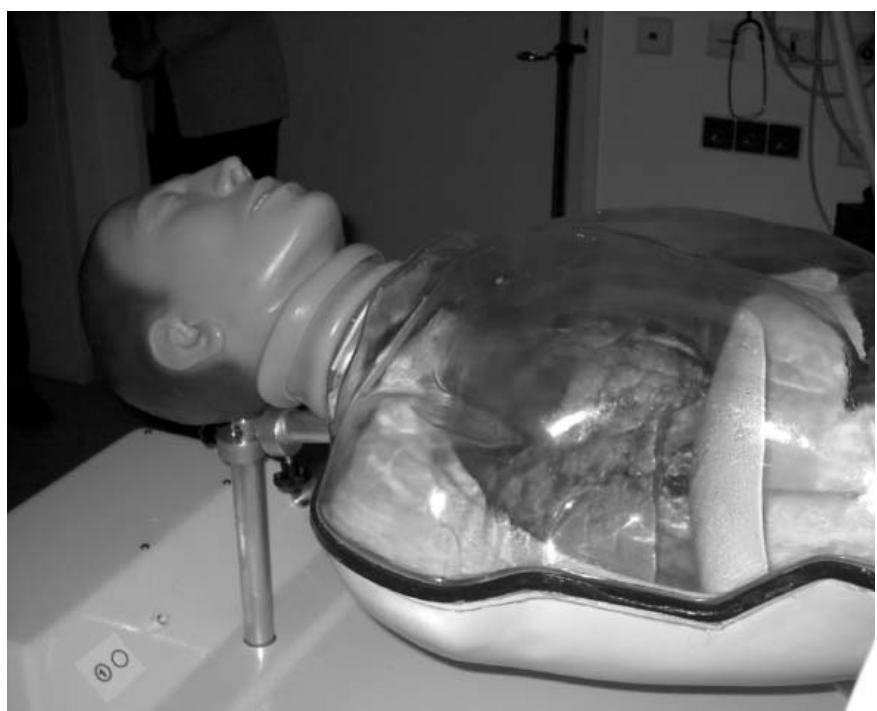

Abb. 3 Prototyp des „Erlanger Broncho-Trainer“ mit umlaufender Dichtung des Modelldeckels und einem Schweinelungenpräparat in einem luftdichten Thoraxraum. Mit einem in der Grundplatte integrierten Vakuumpumpensystem konnte erstmals ein Unterdruck im Thorax erzeugt werden, der die Lunge entfaltet.

die Thoraxhöhle fortgesetzt, durch den dann das Bronchoskop in die biologische Trachea des Lungenpräparates vorgeschoben werden kann. Über diesen gelangt auch die Luft in die angeschlossene biologische Lunge, wobei diese von außen mit einem negativen Druck umgeben wird. Ein weiterer Durchführungsstutzen durch den luftdicht abgeschlossenen Modellkörper dient zum Anschluss des Vakuums, das einen negativen Druck in dem Modell erzeugt und damit die Lunge, entsprechend den physiologischen Verhältnissen, zur Ausdehnung bringt.

\section{Integrierte Vakuumpumpe mit elektronischer Steuerung}

In die Grundplatte des Modells ist eine spezielle Vakuumpumpe integriert, die Saugleistung dieser Vakuumpumpe ist bis zu - $20 \mathrm{~mm}$ Hg einstellbar. Über einen handelsüblichen Saugungsschlauch ist die Saugpumpe mit dem Anschlussstutzen im kaudalen Bereich des luftdicht verschlossenen Modellkörpers verbunden. Nach Einschalten der Vakuumpumpe kann die Luft über den Stutzen im kaudalen Bereich des Modells aus der Bauchhöhle und Thoraxhöhle abgesaugt werden und ein Vakuum im Thorax erzeugt werden. Bei einem Unterdruck von ca. - $15 \mathrm{~mm} \mathrm{Hg}$ dehnen sich beide Lungenflügel komplett aus. Die Saugpumpe ist über einen elektronischen Steuerungsmechanismus so geschaltet, dass intermittierende unterschiedlich lange Inspirations- und Exspirationsphasen resultieren. Mit diesem speziell geschalteten Unterdrucksystem kann sehr eindrucksvoll der Effekt einer „atmenden Lunge“ simuliert werden.

\section{„Atmende Lunge“ - erste Erfahrungen im Rahmen eines Bronchoskopie-Workshops}

Diese neu entwickelte „atmende Lunge“ wurde Ende November erstmals im Rahmen eines Bronchoskopie-Workshops in Gerlingen bei Stuttgart in der Klinik Schillerhöhe unter der Leitung von Prof. Dierkesmann eingesetzt und konnte eindrucksvoll mit erstaunlicher Realitätsnähe überzeugen. Der in der Thoraxhöhle erzeugte Unterdruck reicht aus, um die Lunge beidseits zur vollständigen Entfaltung zu bringen. Die präparierte Lunge war zuvor tief gefroren gelagert und wurde erst für den Workshop aufgetaut. Es hat sich dabei gezeigt, dass der „Surfactant-Faktor“ und die Gewebebeschaffenheit nach Einfrieren des Präparates ausreicht, um eine vollständige Entfaltung zu erreichen. Nach Entfaltung der Lunge wurde die Vakuumpumpe über eine spezielle elektronische Steuerung von permanentem Saugbetrieb auf intermittierenden Saugbetrieb umgestellt. Die Inspirationsund Exspirationsintervalle konnten dabei getrennt eingestellt werden. In der Phase der Exspiration, in der über ein Magnetventil die Absaugung aus der Thoraxhöhle unterbrochen wird, und es zu einem Druckausgleich in dem Modell kommt, kollabierte die Lunge aufgrund der Retraktionskraft. Sobald im Inspirationsintervall die Saugwirkung wieder die Thoraxhöhle evakuiert, entfaltete sich die Lunge wieder. Mit diesen über ein spezielles Vakuumsystem einstellbaren Inspirations- und Exspirationsphasen wird der eindrucksvolle Effekt einer „atmenden Lunge“ erreicht. Auch das echte "Gewebegefühl“ und der natürliche optische Eindruck wurden als ideale Übungsmöglichkeit vor allem für interventionelle Eingriffe gesehen.

Ein aufgetautes Schweinelungenpräparat hielt im „Atmungsbetrieb" die gesamte Dauer des Workshops (4 Stunden) und überstand auch kleinere Perforationen bei der peripheren Biopsie, ohne dass sie kollabierte. Interessanterweise kam es nach Abstellen der Unterdruckbeatmung zu einer schaumigen Blasenbildung in den Bronchien, ähnlich wie man es vom Lungenödem kennt.

\section{Vergleich eines Schweinelungenpräparates mit der menschlichen Anatomie}

Um die bekannten anatomischen Unterschiede des Bronchialsystems vom Schwein im Vergleich zum humanen Bronchialsystem möglichst anschaulich darzustellen, haben wir die Luftwege eines Schweinelungenpräparates mit einem eingefärbten Kunststoffgranulat ausgegossen und nach dessen Aushärtung das Gewebe weggeätzt, so dass der eingefärbte Bronchialbaum mit seinen feinen Aufzweigungen übrigblieb (Abb.4).

Anders als beim Menschen zweigt der rechte Oberlappenbronchus bereits oberhalb der Bifurkation aus der Trachea ab, um sich dann in einen apikalen, einen anterioren und einen posterioren Segmentbronchus aufzuzweigen. Vom rechten Hauptbron- 


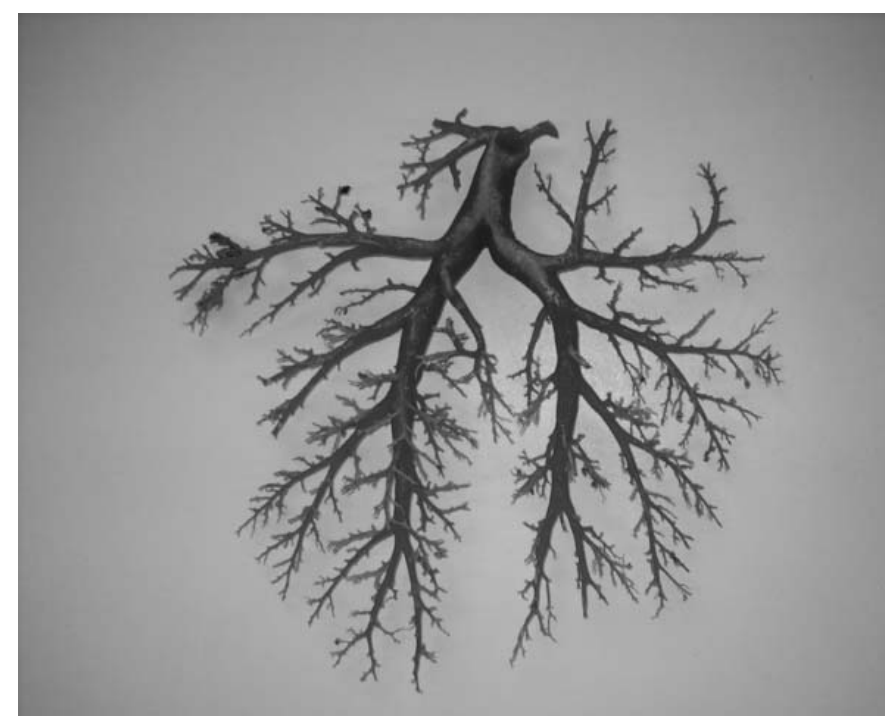

Abb. 4 Eingefärbter Kunststoffausguss eines Bronchialsystems vom Schwein.

chus zweigt der Mittellappenbronchus mit einem medialen und lateralen Segment ab, ein Zwischenbronchus ist nicht ausgebildet. Ein kurzes Stück distal des Mittellappenabgangs zweigt nach dorsal das apikale Unterlappensegment ab und ist mit dem Segment 6 des Menschen vergleichbar. In dieser Höhe zweigt nach ventral/medial der kleine „Lobus accessorius“ ab. Im weiteren Verlauf zweigen dann vom Unterlappenbronchus 3 weitere dorsale und 4 ventrale Abgänge ab. Die Verhältnisse auf der linken Seite sind bis auf die zusätzlichen Unterlappensegmente durchaus mit der menschlichen Anatomie vergleichbar. Unterschiede bestehen vor allem auf der rechten Seite, da hier der Oberlappenbronchus bereits vor der Bifurkation aus der Trachea abzweigt und nach der Abzweigung des Mittellappenbronchus ein atypischer Abgang zu einem kleinen ventral/medial gelegenen accessorischen Lappen vorhanden ist, der beim Menschen nicht existiert [12].

\section{Diskussion}

Die hier erstmals vorgestellte Modifikation des Erlanger EndoTrainers als „Erlanger Broncho-Trainer" mit einer atmenden und vollständig entfaltbaren biologischen Trainingslunge vom Schwein verbessert nicht nur die anatomische Orientierung durch die dreidimensionale Entfaltung der Lunge, sondern bietet auch mit überzeugender Realität eine gute Übungsmöglichkeit für bronchoskopische Interventionen. Bei interventionellen Techniken für Fortgeschrittene kommt es weniger auf die exakte Anatomie an - hier gibt es, wie dargestellt, geringe Abweichungen von der menschlichen Anatomie - sondern viel mehr auf den realistischen und lebensechten Gesamteindruck für den Untersucher, vor allem aber auch auf das „natürliche“ Gewebegefühl.

Bei einem ersten Broncho-Trainer Workshop am 07. 2. 2004 im Klinikum Nürnberg in Kooperation mit Herrn Priv.-Doz. Dr. J. H. Ficker wurde die praktische Schulung der Teilnehmer in den Techniken BAL (bronchoalveoläre Lavage), TBNA (transbronchiale Nadelaspirationsbiopsie) und peripheren Zangenbiopsie unter Durchleuchtungskontrolle sowie Fremdkörperextraktionen und

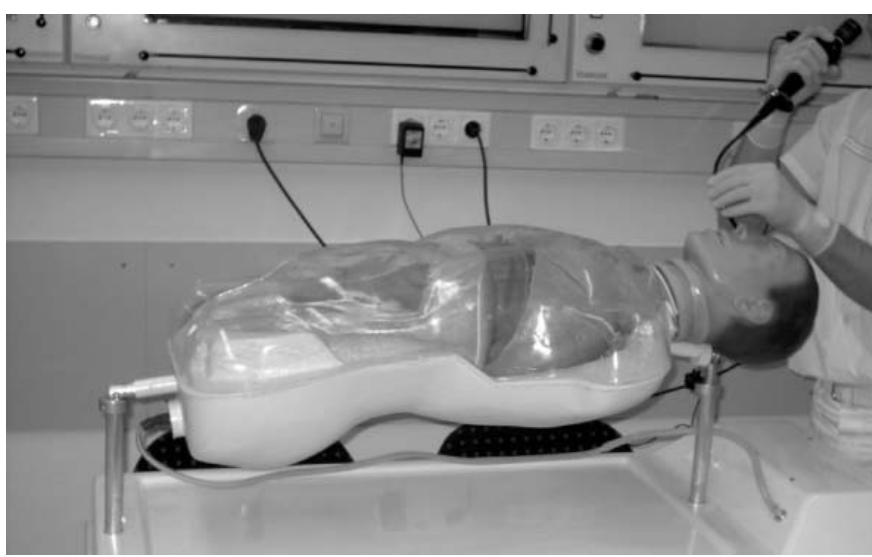

Abb. 5 Bronchoskopie an „atmenden“ Broncho-Trainer mit Vorbereitung einer Broncho-Alveolären-Lavage (BAL).

die Therapie mit dem Argonplasmabeamer erfolgreich erprobt (Abb. 5).

Weitere interventionelle Techniken werden derzeit erprobt, da der besondere Wert des Modells darin liegen könnte, dass neben diagnostischen Techniken auch diverse interventionelle Techniken trainiert werden können wie beispielsweise Fremdkörperentfernungen, Stentimplantationen, Lasertherapie, Therapie mit dem Argonplasmabeamer, Elektrokoagulation, Kryotherapie und Blutstillungstechniken wie Ballonokklusion und Platzierung einer Tamponade.

\section{Literatur}

${ }^{1}$ Neumann M, Hochberger J, Felzmann T et al. The Erlangen Endo-Trainer. Endoscopy 2001; 33: 887-890

2 Neumann M, Hohenberger W. "Score-Card-Endoscopy" - Leistungskontrolle oder Leistungsmotivation? Mitt Dtsch Ges Chir 2003; 2/03: $150-157$

${ }^{3}$ Neumann M et al. Bronchoskopie-Workshop, www.endo-trainer.de/ ece/german/bronchoskopie.php

${ }^{4}$ Ram B, Oluwole M, Blair RL et al. Surgical simulation: an animal tissue model for training in therapeutic and diagnostic bronchoscopy. J Laryngol Otol 1999; 113: 149-151

${ }^{5}$ Wood RE, Pick JR. Model systems for learning pediatric flexible bronchoscopy. Pediatr Pulmonol 1990; 8: $168-171$

${ }^{6}$ Vining DJ, Liu K, Choplin RH et al. Virtual bronchoscopy. Relationships of virtual reality endobronchial simulations to actual bronchoscopic findings. Chest 1996 ; 109: 549-553

${ }^{7}$ Colt HG, Crawford SW, Galbraith 3rd O. Virtual reality bronchoscopy simulation: a revolution in procedural training. Chest $2001 ; 120$ : $1333-1339$

${ }^{8}$ Ost D, DeRosiers A, Britt EJ et al. Assessment of a bronchoscopy simulator. Am J Respir Crit Care Med 2001 Dec 2001; 164: 2248 - 2255

${ }^{9}$ Moorthy K, Smith S, Brown T et al. Evaluation of virtual reality bronchoscopy as a learning and assessment tool. Respiration 2003 ; 70: 195-199

${ }^{10}$ Beamis Jr JF, Shapshay SM, Setzer S et al. Teaching models for Nd:YAG laser bronchoscopy. Chest 1989 ; 95: 1316-1318

${ }^{11}$ Franchella A, Shweiki F, Riccipetitoni G et al. Left lung transplantation in piglets: a training model. PMID: 8108947 [PubMed - indexed for MEDLINE].

12 Nickel R. Lehrbuch der Anatomie der Haustiere, Band II, 6. Auflage. Berlin, 1987: 227-280

${ }^{13}$ Neumann M, Mayer G, Ell C et al. The Erlangen Endo-Trainer: lifelike simulation for diagnostic and interventional endoscopic retrograde cholangiography. Endoscopy 2000; 32: $906-910$ 